Methods: The study population consisted of $15 \mathrm{pSS}$ patients, who fulfilled the ACR-EULAR criteria and underwent salivary gland biopsies of both the parotid and labial gland. Patients were not treated with biologicals and 13 patients were not using other immunosuppressive therapy. Presence and severity of LELs were evaluated on $\mathrm{HE}$ stained sections. Severity of lesions was scored from stage 0 to stage 3 (stage 0: lymphocytic ductal infiltration without hyperplasia of the epithelium; stage 1: Iymphocytic ductal infiltration and $<50 \%$ hyperplastic epithelium; stage 2: Iymphocytic ductal infiltration and $\geq 50 \%$ hyperplastic epithelium; stage 3 : lymphocytic ductal infiltration and fully circumferentially hyperplastic epithelium with occluded lumen). Numbers of B- and T-lymphocytes within LELs were counted (10 ducts per biopsy) by using Image $\mathrm{J}$ cell counter on serial CD20 and CD3 stained sections. High molecular weight cytokeratin staining was used to identify ductal borders. Generalized estimating equations (GEE) were used to analyse the numbers of B- and T-lymphocytes and B/T ratio's over the different stages of severity of LELs.

Results: B- and T-lymphocytes can both infiltrate within the ductal epithelium forming LELs in salivary glands of pSS patients. T-lymphocytes were present in all LELs, scattered through the whole ductal epithelium. Whereas B-lymphocytes were found in clusters, mostly located in the hyperplastic area of the ductal epithelium. With higher severity of LELs, the numbers of B- and T-lymphocytes increased significantly, in both the parotid and labial gland. The numbers of B-lymphocytes increased relatively more with higher severity of LELs than T-lymphocytes. This has led to an increased intraepithelial $\mathrm{B} / \mathrm{T}$ ratio in more pronounced LELs. This increased $B / T$ ratio was even more pronounced in parotid than in labial glands. In both glands, there was a predominance of T-lymphocytes in lower LEL stages. In more severe LEL stages, B-lymphocytes outnumbered the Tlymphocytes.

Conclusions: Given the relative increase in the number of B-lymphocytes with higher severity of LELs as well as their close association with proliferating ductal epithelial cells, we conclude that B-lymphocytes play a major role in the hyperplasia of the ductal epithelium.

Disclosure of Interest: None declared

DOI: 10.1136/annrheumdis-2018-eular.6637

\section{AB0165 RANK/RANK-LIGAND INTERACTION REGULATES PATHOGENIC T CELL RECRUITMENT IN SJÖGREN'S SYNDROME}

S. Nayar ${ }^{1}$, B.A. Fisher ${ }^{1}$, J. Campos ${ }^{1}$, D.H. Gardner ${ }^{1}$, A. Dumusc ${ }^{2}$, V. lannizzotto ${ }^{1}$, C. Smith ${ }^{1}$, C.D. Buckley ${ }^{1}$, S.J. Bowman ${ }^{3}$, C.G. Mueller ${ }^{4}$, F. Barone' ${ }^{1}$.

${ }^{1}$ Rheumatology Research Group, Institute of Inflammation and Ageing, University of Birmingham, Birmingham, UK; ${ }^{2}$ rheumatology department, Departement de l'appareil locomoteur, Lausanne University Hospital (CHUV), Lausanne, Switzerland; ${ }^{3}$ University Hospitals Birmingham National Health Service Foundation Trust, Birmingham, UK; ${ }^{4}$ Institut de Biologie Moleculaire et Cellulaire, Strasbourg, France

Background: The RANKL (ligand)-RANK-OPG triad, members of the TNF(R) superfamily, is implicated in lymphoid organ development and bone homeostasis It has recently been demonstrated that RANK-activated astrocytes release CCL20 and attract T cells to the central nervous system in a model of Multiple Sclerosis and that transgenic RANK expression in the skin promotes aberrant epithelial cell proliferation and is sufficient to induce ectopic formation of tertiary lymphoid structures (TLS). Ductal epithelial cells (SGEs) have been implicated in Sjögren's Syndrome (SS) pathogenesis where they mediate immune recruitment by expression of pro-inflammatory chemokines and support the formation of premalignant myoepithelial lesions.

Objectives: A combination of human and mouse studies were used to address the role of RANK-RANKL interaction in primary $(p)$ SS

Methods: Salivary glands (SGs) and saliva samples from patients recruited in the OASIS cohort (University of Birmingham) were studied to evaluate this pathway in human disease. Consecutive stimulated saliva samples $(n=69)$ were analysed using Proseek Multiplex INF ${ }^{96} \times 96$, covering 92 unique inflammation-related protein biomarkers. Taking advantage of a viral induced model of pSS we studied the effect of this pathway with a RANKL blocking antibody and by inducing gain of function with direct cannulation in the salivary glands of recombinant RANKL. Murine SGs were studied by immunofluorescence, flow cytometry and qPCR on total tissue and sorted cells.

Results: Fourteen proteins in saliva were significantly separated between pSS and sicca controls, and elevated levels of just two proteins, RANKL and TNF $\beta$, could classify pSS or sicca with $75 \%$ accuracy. Levels of salivary RANKL and CCL20 were strongly correlated $(r=0.6 ; p<0.01)$. We demonstrated that both human and murine inflamed SGEs upregulate both RANK and CCL20, a chemokine known to recruit pathogenic T cells. Upregulation of RANKL was found in human Th2 cells, classically associated with humoral responses and germinal centre (GC) formation. SGs from mice treated with anti-RANKL antibody showed decreased epithelial proliferation, reduced $T$ cell infiltration and defective TLS establishment. On the contrary, viral infected SGs treated with recombinant RANKL showed increased T cell infiltration, CCL20 expression and enhanced differentiation of GC B cells.

Conclusions: In vivo RANK/RANKL interaction mediates recruitment of activated T cells that are skewed toward a Th2 phenotype. These, in turn, will favou the establishment of TLS in the SG. Those data were confirmed in human pSS where expression of RANK is found in inflamed epithelium and RANKL detection in saliva is able to differentiate patients with pSS from sicca controls, thus candidates this pathway both for drug targeting and patient stratification.

Disclosure of Interest: None declared

DOI: 10.1136/annrheumdis-2018-eular.6563

\section{AB0166 TRACKING OF MUCOCUTANOUS AND MUSCULOSKELETAL FLARES IN SLE USING SERUM FAS, FERRITIN, IGFBP2 AND STNFRII}

S. Soliman ${ }^{1,2}$, K. Vanarsa ${ }^{2}$, A. Swilling ${ }^{2}$, J. Merrill ${ }^{3}$, C. Mohan ${ }^{2} .{ }^{1}$ Rheumatology and Rehabilitation, Faculty of Medicine, Minia University hopsital, Minya, Egypt, ${ }^{2}$ biomedical engineering, university of Houston, Houston; ${ }^{3}$ Oklahoma Medical research foundation, Oklahoma, United States

Background: SLE is a multisystemic autoimmune disease characterized by unpredictable disease course with periods of flares and remission. The lack of reliable methods which can predict a disease flare hampers the exploration of effec tive and preventive strategies for disease relapses.

Objectives: To study the performance of serum FAS, sTNFRII, Igfbp2 and Ferritin as biomarkers for tracking lupus flares in non-renal SLE patients.

Methods: Twenty-nine patients, who met the requirements of American College of Rheumatology (ACR) for classification of SLE, were recruited from Oklahoma Medical Research Foundation (OMRF) for serological testing of all four serum protein markers. None of the patients had lupus nephritis. Serum samples were obtained over 4 consecutive visits. Serum FAS, sTNFRII, Igfbp2 and Ferritin molecules were measured in all patients. Lupus disease activity was assessed by both SLEDAI and BILAG activity indices.

Results: In our study, all 4 tested biomarkers (FAS, Ferritin, Igfbp2 and sTNFRII) showed significant correlations with SLEDAI and BILAG. FAS $(r=0.36, p<0.0001$ for SLEDAI \& $r=0.29, p=0.0002$ for BILAG), Ferritin $(r=0.13, p=0.0494$ for SLEDAI \& $r=0.22, p=0.0035$ for BILAG), Igfbp2 $(r=0.24, p=0.0013$ for SLEDAI \& $r=$ $0.18, p=0.0106$ for BILAG) and sTNFRII $(r=0.30, p<0.0001$ for SLEDAI \& $r=0.19$ $p=0.0112$ for BILAG). When serial disease activity changes were examined, dif ferent serum markers performed better in different SLE patients, tracking with mucocutaneous or musculoskeletal disease flares. In studying a total of 72 dis ease intervals, FAS and Ferritin exhibited the highest concordance with concurrent disease activity (58-62\%), followed by lgfbp2 (50\%) and sTNFRII (46\%), all of which were superior to the performance of complement C3, C4 and anti-DNA. Furthermore, adding FAS to other tested molecules increased its ability to track concordant disease activity changes (81\% for FAS $\pm F$ Ferritin; $76 \%$ for FAS \pm Igfbp2 and FAS \pm STNFRII).

Conclusions: Serum FAS and Ferritin emerge as potential serum markers for tracking mucocutaneous or musculoskeletal disease flares in SLE patients.

Disclosure of Interest: None declared

DOI: 10.1136/annrheumdis-2018-eular.1133

\section{\begin{tabular}{|l|l}
\hline AB0167 METFORMIN HAS ANTI-INFLAMMATORY POTENTIAL BY \\
\hline
\end{tabular} REDUCING P-GP EXPRESSION ON PBMCS OF PATIENTS WITH LUPUS}

S. Kansurkar, M.K. Rai, D.P. Misra, V. Agarwal. Clinical Immunology and Rheumatology, Sanjay Gandhi Postgraduate Institute of Medical Sciences (SGPGI), Lucknow, Uttar Pradesh, India, Lucknow, India

Background: Metformin causes immunomodulation by activation of AMP-kinase and thereby inhibition of mTOR pathway. Metformin reduces disease activity in lupus mouse models. ${ }^{1}$ A study has shown that its use in lupus patients decreased corticosteroid dose and prevented flares. ${ }^{2}$ P-glycoprotein expression is linked to drug resistance and increased cytokine production. Metformin inhibits expression of $\mathrm{P}$ - Glycoprotein (P-gp) in cancer cells. ${ }^{3}$ There is scarce data on effect of metformin on P-gp expression and cytokine secretion on immune cells in autoimmune diseases like lupus.

Objectives: To study the effect of metformin on P-gp expression in PBMCs of lupus patients and its effect on inflammatory cytokines secretion. To determine optimum concentration required for such an effect.

Methods: PBMCs of nine lupus patients(Mean age $30 \mathrm{yrs}$, all females) were cultured using RPMA medium and then stimulated with PMA/ ionomycin, with or without increasing dose of metformin $(0.01,0.1,1,10 \mathrm{mMol} / \mathrm{L})$ for $24 \mathrm{~h}$. Cytokines IL$1 \mathrm{~b}, \mathrm{IL}-6, \mathrm{IFN}-\mathrm{g}$ and IL-10 were analyzed by ELISA in culture supernatant. Cell 
viability was independently assessed by MTT assay. P-gp expression was measured in same samples by flow-cytometry.

Results: In MTT assay viability of cells was maintained across all concentrations of metformin used in this study(figure 1a). Metformin decreased expression of P$\mathrm{gp}$ in PBMCs in dose dependent manner ( $p=0.003)$. (figure $1 b)$. In the PBMC cultures with PMA and increasing concentration of metformin, there was decrease in production of pro-inflammatory cytokines: IL-1b $(p=0.001), \mathrm{IL}-6(\mathrm{p}=0.007)$ and IFNg $(p<0.001)$ at even the lowest concentration of metformin. There was increase in production of anti-inflammatory cytokine IL-10 $(\mathrm{p}=0.014)$. The suppression of IL-1, IL-6, IFNg and increase in IL10 production was dose dependent.
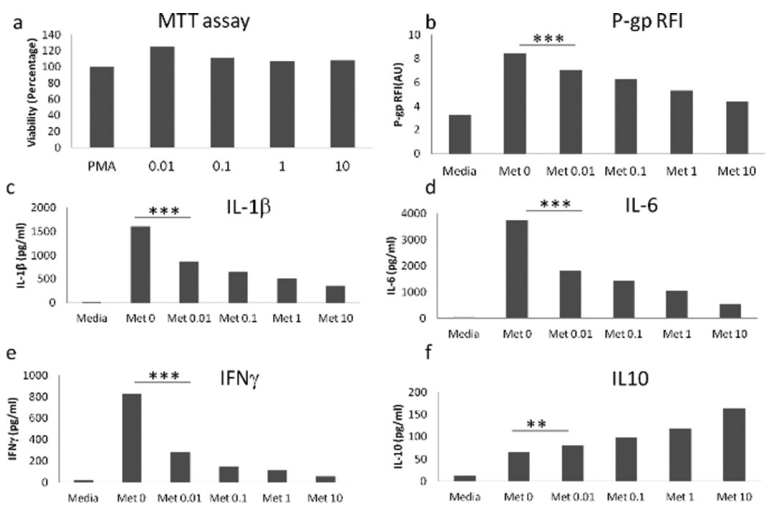

Abstract AB0167 - Figure 1. a: MTT assay for cell viability (ns) b: P-gp expression with increasing metformin concentration (shown on $X$ axis in $\mathrm{mMOL} / \mathrm{L}$ ), c,d,e,f: cytokine alnalyses of IL-1 $\beta, I L-6, I F N-\gamma$ and IL-10 resp. In all experiments, PBMCs were stimulated by PMA and Metformin. ${ }^{* \star *}=\mathrm{P}<0.01,{ }^{* *}=\mathrm{P}<0.03$

Conclusions: Metformin inhibits P-gp expression which is responsible for resistance to action of various drugs including corticosteroids which are cornerstones of treatment in SLE. Metformin thus may help to reduce corticosteroid dose. Antiinflammatory activity seen in this study is occurring at concentrations which are therapeutically achievable. Plasma levels of metformin at the therapeutic doses commonly used for diabetes are usually around $0.01-0.04 \mathrm{mM} / \mathrm{L} .{ }^{4}$ Present study has demonstrated anti-inflammatory effect of metformin at this concentration. Metformin offers dual advantage which has anti-inflammatory activity and also has a potential to reduce drug resistance to other therapeutic agents.

\section{REFERENCES:}

[1] Lee S-Y, Moon S-J, Kim E-K, et al. Metformin Suppresses Systemic Autoimmunity in Roquinsan/san Mice through Inhibiting B Cell Differentiation into Plasma Cells via Regulation of AMPK/mTOR/STAT3. The Journal of Immunology Author Choice 2017;198(7):2661-2670.

[2] Yin Y, Choi SC, Xu Z, Perry DJ, Seay H, Croker BP, et al. Normalization of CD4+ T cell metabolism reverses lupus. Sci Transl Med 2015.

[3] Kim HG, Hien TT, Han EH, Hwang YP, Choi JH, Kang KW, et al. Metformin inhibits P-glycoprotein expression via the NF-kappaB pathway and CRE transcriptional activity through AMPK activation. British journal of pharmacology. 2011;162(5):1096-108.

[4] Ling He, Fredric E. Wondisford, Metformin Action: Concentrations Matter, Cell Metabolism 2015;21(2):159-162.

Acknowledgements: Thanks to Swati Chouhan, Abhishek, Sakir, Rutviz, Suvrat, Pravin, Sonia for their immense help.

Disclosure of Interest: None declared

DOI: 10.1136/annrheumdis-2018-eular.7261

\section{AB0168 ELEVATED SERUM LEVELS OF HMGB1 AND SRAGE IN PATIENTS WITH ANTIPHOSPHOLIPID SYNDROME}

S. Truglia' ${ }^{1}$, V. Manganelli ${ }^{2}$, A. Capozzi ${ }^{2}$, C. Alessandri ${ }^{1}$, E. Lococo ${ }^{2}$, T. Garofalo², M. Sorice ${ }^{2}$, A. Longo ${ }^{2}$, R. Misasi ${ }^{2}$, F. Conti ${ }^{3}$, G. Valesini ${ }^{1} .{ }^{1}$ Dipartimento di Medicina Interna e Specialità Mediche; ${ }^{2}$ Department of Experimental Medicine, Sapienza University; ${ }^{3}$ Dipartimento di Medicina Interna e Specialità Mediche, POLICLINICO UMBERTO 1, Rome, Italy

Background: Antiphospholipid syndrome (APS) is a systemic autoimmune disease characterised by the presence of at least one clinical event among vascular thrombosis and/or pregnancy morbidity, in the presence of circulating antiphospholipid antibodies (aPL). High-mobility group box-1 (HMGB1) is a non-histonic protein belonging to the family of alarmins. It is associated with chromatin and has a dual function depending on the cell state: in basal conditions it is located in the nucleus and promotes the interaction of some transcription factors with DNA, in inflammatory conditions it is secreted in the extracellular space and exerts the functions of a pro-inflammatory cytokine. One of the main receptor system responsible for the HMGB1 activity is the "receptor for advanced glycation end products" (RAGE). Increased serum HMGB1 levels have been reported in patients with Sys temic Lupus Erythematosus and pre-eclampsia, as other alarmins are increased in patients with early abortions.

Objectives: To evaluate the serum levels of HMGB1 and soluble RAGE (sRAGE) in patients with obstetric and thrombotic APS.

Methods: 43 consecutive patients with APS, diagnosed according to the Sapporo criteria, were enrolled. The study cohort included both primary APS $(=15)$ and APS associated with SLE $(=28)$. In addition, 30 healthy subjects $(\mathrm{HC})$ matched for age and sex were studied as controls. Serum levels of HMGB1 and SRAGE were analysed by Western blot.

Results: The clinical features of the enrolled patients (40 females and 3 males, mean age $40.98 \pm 13.48$ years, ) are reported in table 1 . HMGB1 and SRAGE serum levels were significantly increased in APS patients in comparison with controls $(p<0.001)$ (figure 1). Furthermore, no difference in HMGB1serum leved were detected among patients with thrombotic or obstetric APS and patients with primary or secondary APS.APS patients with thrombosis showed higher levels of HMGB1 than APS patients without thrombosis; in addition, in APS patients there is a correlation between HMGB1 serum levels and thrombosis.

Abstract AB0168 - Table 1. Clinical characteristics of APS patients.

Characteristics $n(\%) \quad$ APS

$(\mathrm{n}=43)$

\begin{tabular}{lc}
\hline Vascular thrombosis & $39(90.7)$ \\
Venous thrombosis & $26(60.5)$ \\
Arterial thrombosis & $18(41.9)$ \\
Recurrent thrombosis & $15(34.9)$ \\
Pregnancy morbidity & $15 / 40(37.5)$ \\
Normal fetus deaths & $2(5)$ \\
Premature births & $1(2.5)$ \\
Spontaneous abortions & $13(32.5)$ \\
Vascular thrombosis and Pregnancy morbidity & $11(27.5)$ \\
Non-criteria APS features & \\
Livedo reticularis & \\
Thrombocytopenia & $16(37.2)$ \\
Migraine & $11(25.6)$ \\
Seizures & $11(25.6)$
\end{tabular}

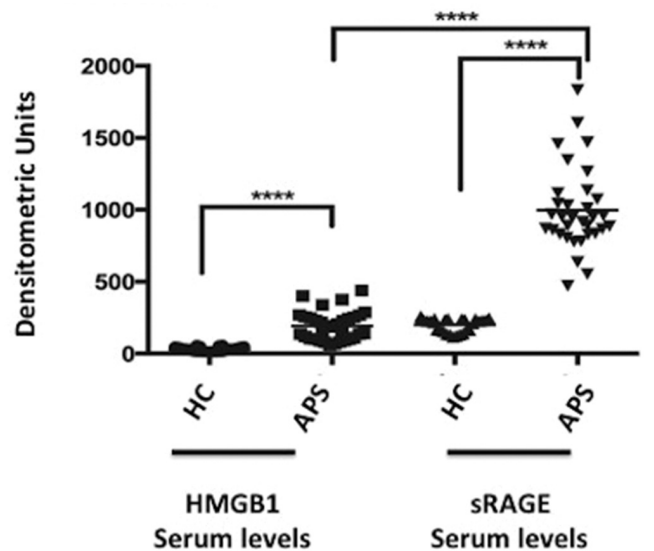

Abstract AB0168 - Figure 1. Serum levels of HMGB1 and SRAGE in APS patients and controls.

Conclusions: In this study, we investigated for the first time the serum levels of HMGB1 and SRAGE in patients with APS, showing increased levels in both primary and secondary APS compared to controls. Larger studies are needed to assess whether monitoring serum HMGB1/sRAGE levels could be a useful tool for risk stratification in patients with APS.

Disclosure of Interest: None declared

DOI: 10.1136/annrheumdis-2018-eular.6187 